\title{
The test-negative design for estimating influenza vaccination effectiveness
}

\author{
Benjamin J. Cowling ${ }^{\star 1}$ and Sheena G. Sullivan ${ }^{2,3}$
}

${ }^{1}$ School of Public Health, The University of Hong Kong, Pokfulam, Hong Kong; ${ }^{2}$ WHO Collaborating Centre for Reference and Research on Influenza at the Peter Doherty Institute for Infection and Immunity, Melbourne, VIC, Australia; ${ }^{3}$ Fielding School of Public Health, University of California, Los Angeles, CA, USA

\section{Objective}

We aimed to describe the theoretical basis and the potential applications of the test-negative design for estimating influenza vaccination effectiveness in sentinel influenza surveillance.

\section{Introduction}

The test-negative design is a variation of the case-control study, in which patients are enrolled in outpatient clinics (and/or hospitals) based on a clinical case definition such as influenza-like illness (ILI). Patients are then tested for influenza virus, and VE is estimated from the odds ratio comparing the odds of vaccination among patients testing positive for influenza versus those testing negative, adjusting for potential confounding factors. The design leverages existing disease surveillance networks and as a result, studies using it are increasingly being reported.

\section{Methods}

We sought to examine the theoretical basis for this design using causal analysis including directed acyclic graphs. We reviewed studies that used this design and examined the study populations and settings, the methodologic choices including analytic approaches, and the estimates of influenza VE provided. We conducted simulation studies to examine specific potential biases.

\section{Results}

We show how studies using this design can avoid or minimize bias, and where bias may be introduced with particular study design variations. A purported advantage of the test-negative design is to minimise selection bias by health-care seeking behaviour and we demonstrate why residual bias may occur. Another purported advantage of the test-negative design is minimization of misclassification of the exposure; however we show how this source of bias may persist and how exposure misclassification may be a greater cause for concern not dealt with by the study design. In our review, we found great variation in estimates, but consistency between interim and final VE estimates from the same locations, and consistency between VE estimates from inpatient and outpatient studies in the same locations, age groups and years. One outstanding issue is the potential bias due to non-collapsibility.

\section{Conclusions}

Our work provides a starting point for further consideration of the validity of the test-negative design, which is an efficient approach for routine monitoring of influenza VE that can be implemented in existing surveillance systems without substantial additional resources. Harmonization of analytic approaches may improve the potential for pooling VE estimates.

\section{Keywords}

influenza; VE; sentinel

\section{*Benjamin J. Cowling}

E-mail: bcowling@hku.hk 